\title{
Mitral valve anatomy: pre-procedural screening and imaging techniques
}

Ariana González-Gómez, MD; Sara Fernández-Santos, MD; Covadonga Fernández-Golfín, MD;

Jose L. Zamorano*, MD, PhD

University Hospital Ramón y Cajal, Madrid, Spain

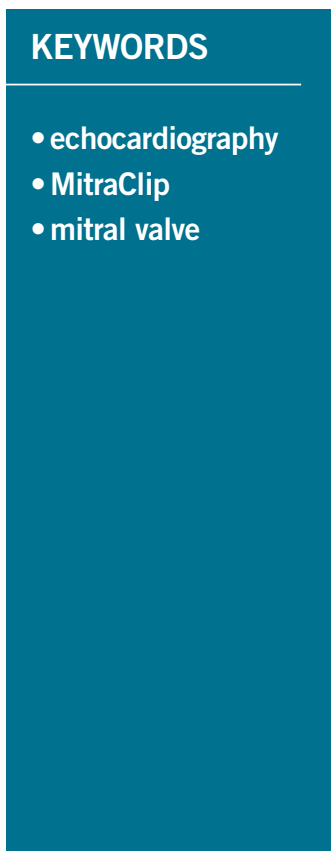

\section{Abstract}

Mitral regurgitation is a source of significant morbidity and mortality with growing prevalence in an increasingly ageing population. The development of reparative percutaneous techniques allows offering treatment to patients ineligible for surgery. The mitral valve is a complex structure and these procedures are limited by anatomical variability and technical complexity. The most established mitral valve repair technique currently is the MitraClip, which requires a careful preoperative evaluation of the mitral valve apparatus to assess the patient's suitability for the intervention, where echocardiography plays an essential role.

\footnotetext{
*Corresponding author: University Hospital Ramón y Cajal, Carretera de Colmenar Km 9.400, 28034 Madrid, Spain. E-mail: zamorano@secardiologia.es
} 


\section{Introduction}

Mitral regurgitation (MR) is increasingly prevalent in developed countries and is a significant cause of morbidity and mortality ${ }^{1}$. Even though open heart surgery is the treatment of choice for these patients, many are denied surgery due to advanced age, LV dysfunction or other comorbidities. This has led to the development of percutaneous mitral valve (MV) procedures as an alternative to open heart surgery in selected patients.

The MV apparatus is a complex anatomical and functional structure, comprising several components (MV annulus, mitral leaflets and subvalvular apparatus), which, along with the left ventricle (LV) and atrium (LA), are essential for integrity and proper valve function. Patient selection and procedural performance of percutaneous valve repair techniques, such as direct leaflet repair, direct or indirect annuloplasty or ventricular remodelling, require a correct and detailed understanding of the MV apparatus and its surrounding structures for procedural success ${ }^{2}$. Echocardiography in two and three dimensions plays an essential role in the morphologic and functional assessment of the MV, allowing the selection of patients who can benefit from percutaneous interventions.

The aim of this review is to describe the MV anatomy and its influence on pre-procedural screening for MV percutaneous interventions.

\section{Anatomy of the mitral valve}

The MV has two leaflets, named anterior and posterior, attached at its base to the mitral annulus and to the subvalvular apparatus from its free edges by means of the chordae tendinae. The anterior leaflet has a trapezoidal shape and occupies one third of the annular circumference. The posterior mitral leaflet, quadrangularshaped, takes up the remaining two thirds of the annulus. In order to ease diagnosis and communication, both leaflets are divided into three individual scallops, although only the posterior leaflet has two real anatomical indentations which visibly define its three portions, labelled P1, P2 and P3. The two commissures are the areas where the two leaflets are connected: the anterolateral commissure, which joins A1 and P1 and is located in the vicinity of the left atrial appendage and the posteromedial commissure, at the junction of A3 and P3 and positioned adjacent to the interventricular septum and the tricuspid valve.

The tissue that connects the LA, LV and mitral leaflets is the MV annulus. It is an oval and three-dimensional structure with a saddle-shaped appearance ${ }^{3}$ with pronounced size changes through the cardiac cycle. During systole, the MV annulus area is reduced by up to $25 \%$, contributing to optimal coaptation of both leaflets.

The anterior portion of the MV annulus is continuous with the aortic annulus, representing the highest (more atrial) part of the annulus. The posterior segment of the MV annulus is more loosely anchored to its surrounding tissue: the lack of a fibrous structure, as opposed to the aortic mitral curtain, explains why annular dilatation and calcification occur here in a great proportion of patients ${ }^{4}$.

The chordae tendinae and the papillary muscles comprise the subvalvular apparatus. The tendinous cords are fibrous strings originating in the papillary muscles and inserted into the mitral leaflets. There are two papillary muscles, originating from the apical third of the LV and labelled in consonance with their anatomical relationship with the mitral commissures. The anterolateral papillary muscle receives dual blood supply from the anterior descending and circumflex coronary arteries, whilst the posteromedial papillary muscle is irrigated only by circumflex or right coronary artery branches, which explains its higher susceptibility to ischaemia.

Finally, the LV also plays an important role in MR pathophysiology due to its close relationship with the papillary muscles. LV remodelling or dilatation, due to ischaemic or non-ischaemic cardiomyopathies, can produce tethering and displacement of papillary muscles reducing mitral leaflet coaptation and generating functional MR (Figure 1).

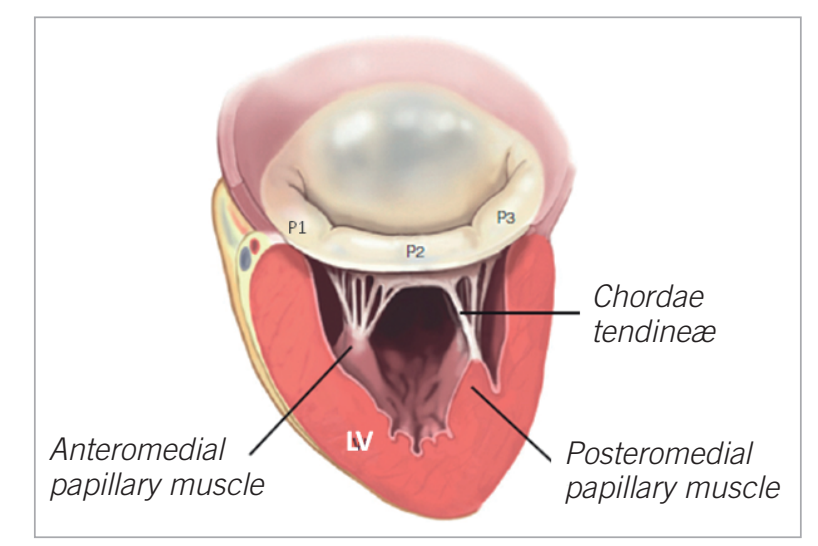

Figure 1. Anatomy of the mitral valve apparatus. LV: left ventricle Published with permission of the author and the publisher. Original source: Castillo JG, Solis J, González-Pinto A, Adams DH. Surgical Echocardiography of the Mitral Valve. Rev Esp Cardiol. 2011;64:1169-81.

\section{Mitral valve percutaneous repair techniques: pre-procedural screening ANNULOPLASTY PROCEDURES}

Effective and durable results after surgery require annuloplasty as a key step. Several percutaneous techniques have been developed mimicking surgical annular remodelling. These techniques are aimed mainly at patients with Type I (incomplete coaptation due to annular dilation/deformation), or Type IIIb (restricted leaflet motion during systole caused by LV enlargement with apical papillary muscle displacement) MR according to Carpentier's classification.

Transcatheter annuloplasty techniques can be performed indirectly via the coronary sinus/great cardiac vein or by approaching the annulus directly via the LA or LV.

Indirect annuloplasty techniques with a transcoronary venous approach use the anatomical relationship between the mitral annulus and the venous system of the heart. The coronary sinus/great cardiac vein surrounds the posterior mitral annulus. A shortening device is inserted into the coronary sinus, indirectly affecting the 
posterior mitral annulus geometry, in an attempt to imitate the effect of a ring in a surgical annuloplasty and to reduce the septallateral diameter ${ }^{5}$.

Cardiac multi-detector computed tomography (MDCT) can provide information on the feasibility of these techniques, since several anatomical features should be taken into consideration. In the first place, there is substantial variability in the distance between the coronary sinus and the mitral annulus, and this distance may be increased with annular dilatation, mainly in the posterolateral position. Another concern of indirect annuloplasty is the risk of coronary ischaemic events due to the close but variable relationship between the coronary sinus and the left circumflex artery ${ }^{6}$.

Direct annuloplasty techniques use a direct access to the mitral annulus, using the retrograde (via the aorta into the LV) or the transseptal approach, in an attempt to reproduce surgical annuloplasty more closely and to avoid the potential limitations of indirect annuloplasty. However, they are technically more complex, and annular calcification, the potential for leaflet damage and circumflex artery damage are of concern. Other techniques such as energy-mediated annuloplasty have also been attempted.

\section{LEAFLET REPAIR}

The main goal of leaflet procedures is to improve leaflet coaptation and reduce the regurgitant orifice. Currently, the most advanced and the most used percutaneous technology for MV repair is the MitraClip ${ }^{\circledR}$ system (Abbott Vascular, Abbott Park, IL, USA). This catheter-based technique implants a clip that holds the free edges of the mid portions of the anterior and posterior mitral leaflets together, creating a double orifice, mimicking the edge-toedge Alfieri surgical technique. The MitraClip procedure has been shown to be a feasible and safe alternative for patients ineligible for surgery ${ }^{7}$.
Echocardiography is the main imaging technique used for patient selection, and is also essential during the intervention, evaluation of results and detection of complications. The procedure is technically demanding, and fluoroscopy alone is not enough for guiding, since the MV leaflets are not seen, and continuous echocardiography imaging is required. Three-dimensional echocardiography (3DE) complements two-dimensional echocardiography (2DE), providing valuable additional information, and should be used when available (Figure 2).

\section{PATIENT SELECTION}

Along with clinical characteristics, patient selection for MitraClip implantation should be based on the evaluation of the severity of MR, the aetiology and mechanism of MR and several anatomical premises.

\section{Mitral regurgitation severity}

Echocardiography is the imaging technique best suited for a comprehensive assessment of MR severity. Initial evaluation is usually performed using transoesophageal echocardiography (TOE). TOE is mandatory for the evaluation of potential candidates for MitraClip implantation since it provides higher accuracy. An intervention should only be indicated in cases of moderate to severe or severe MR.

With 2D TOE, the PISA (proximal isovolumetric surface area) method is most commonly used to calculate the effective regurgitant orifice area (EROA) and the regurgitant volume (RV). In primary $\mathrm{MR}$, severe $\mathrm{MR}$ is defined by an EROA $>40 \mathrm{~mm}^{2}$ and an $\mathrm{RV} \geq 60 \mathrm{ml}$. In functional MR, lower thresholds are used (EROA $>20 \mathrm{~mm}^{2}$ and an $\mathrm{RV} \geq 30 \mathrm{ml}$ ). 3D TOE adds value to 2D imaging in the evaluation of MR severity. From a 3D colour Doppler data set, after reformatting with orthogonal axis alignment, perpendicular to the jet, an en face view of the regurgitant jet can be obtained. Manual planimetry of the vena contracta area can be performed without any geometric assumptions with high accuracy

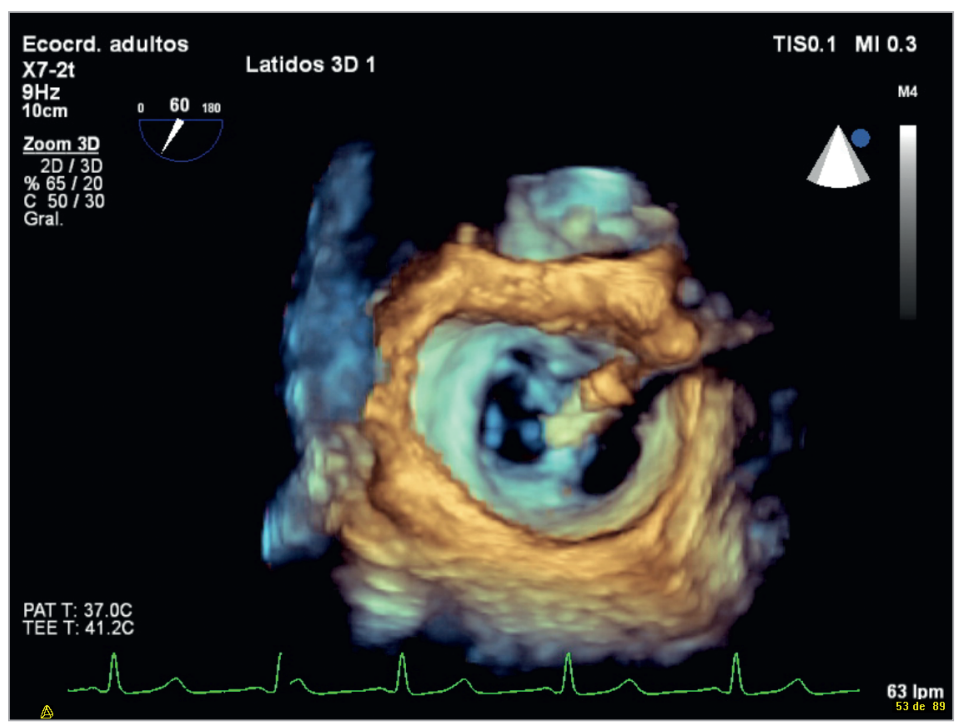

Figure 2. 3D transoesophageal echocardiography. MitraClip holding the free edges of the mid portions of the anterior and posterior mitral leaflets together, creating a double orifice. 
independently of eccentricity or jet morphology. However, despite the different methods available, no single method is good enough to establish MR severity in the majority of patients, and an integrated approach is recommended ${ }^{3,8}$ (Figure 3 ).

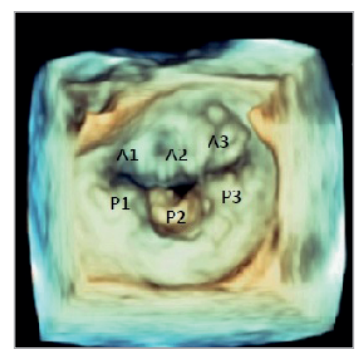

Figure 3. 3D transoesophageal echocardiography. The mitral valve from the left atrial perspective, showing P2 mitral valve prolapse.

\section{Aetiology and mechanism of mitral regurgitation}

Patients eligible for MitraClip implantation are those with organic valvular lesions secondary to degenerative MV prolapse, or functional/ischaemic MR (secondary MR). Other aetiologies contraindicate the procedure.

A detailed evaluation of MR aetiology and mechanism should be performed using TOE before the procedure. 3DE complements 2DE, allowing the visualisation of the whole MV in a single en face view from the LA perspective (the so-called surgeon's view) or from the LV. This provides a more accurate identification of prolapsing scallops or leaflet abnormalities and allows localisation of MR jet origin and morphology (more hemi-elliptical than hemispheric in some cases, especially in those of functional MR) (Figure 3).
Identifying central or eccentric jets is important. Central jets are the ones that have shown the best results, since the MitraClip system creates a double orifice at the level of A2-P2. In order to ensure a safe implant, the degree of thickness and calcification of mitral leaflets should be assessed as well, since the presence of significant calcification especially in the grasping area makes the patient unsuitable for the procedure (Figure 4).

\section{Criteria of eligibility for MitraClip implantation}

Several anatomical criteria that make a patient eligible for MitraClip implant were defined in EVEREST ${ }^{7}$. As mentioned before, the presence of central jets is preferred, and leaflet tissue quality should be assessed.

For patients with degenerative MV prolapse, two anatomical measurements must be evaluated. The flail gap (distance separating the tip of the flail segment from its opposing normally coapting leaflet) measured by 2D TOE in LVOT and four-chamber views should be $<10 \mathrm{~mm}$. The flail width derived from the intercommissural 2D TOE view and/or transgastric short-axis view should be $<15 \mathrm{~mm}$.

For patients with functional MR, a coaptation length measured in the four-chamber view and LVOT view of at least $2 \mathrm{~mm}$, and a coaptation depth below the mitral annular plane on a four-chamber view no more than $11 \mathrm{~mm}$ must be available. The length of the posterior and anterior leaflets is also required (Figure 5).

The MV area must be more than $4 \mathrm{~cm}^{2}$ in order to avoid mitral stenosis. 3D TOE can be useful for the determination of the MV area. Another important issue to assess is the dimension of the LA and interatrial septal morphology, with the presence of patent foramen ovale depicted. Increasing experience has shown that the MitraClip device can be successfully implanted in patients with a more complex MV anatomy. The presence of sufficient leaflet tissue for mechanical coaptation constitutes the most important factor.

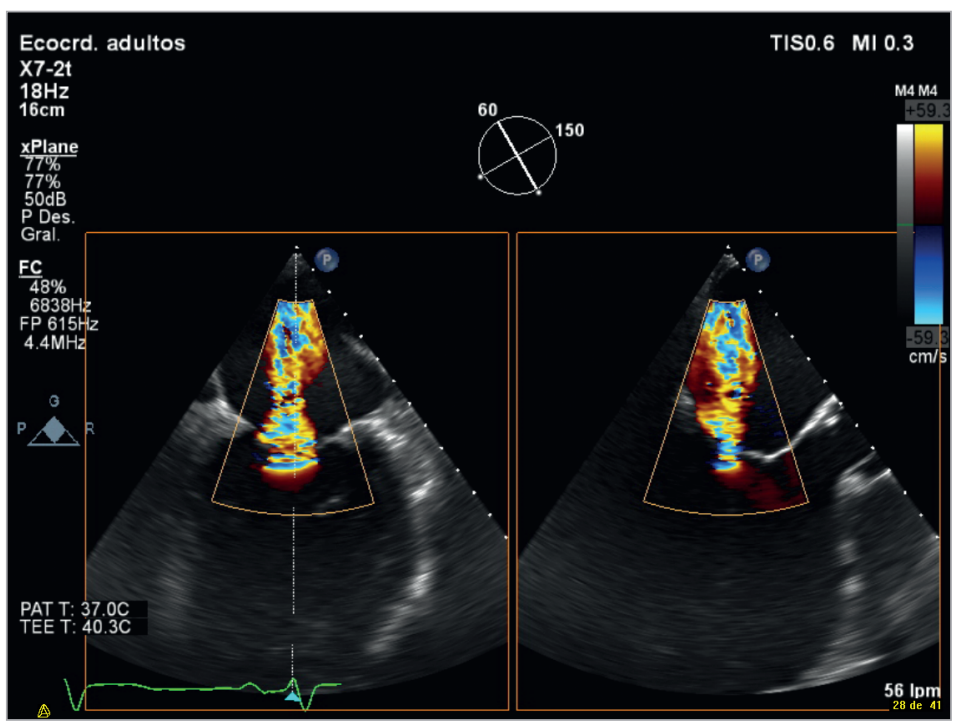

Figure 4. 2D transoesophageal echocardiography. Two simultaneous orthogonal views showing a central jet in a patient with severe functional mitral regurgitation. 


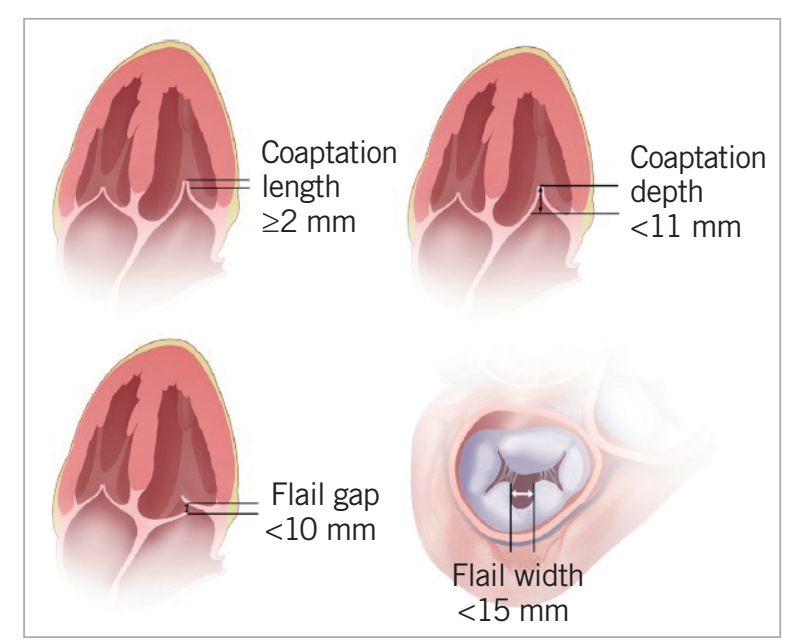

Figure 5. Key anatomical eligibility criteria for MitraClip implant.

\section{Other techniques}

Other percutaneous techniques have been developed, such as the iCoapsys $^{\mathrm{TM}}$ system (Myocor, Maple Grove, MN, USA), based on the Coapsys surgical procedure. This approach attempts to reduce the septal-lateral ventricular distance to improve leaflet coaptation by implanting two epicardial pads on both sides of the LV among which a transventricular bridge is lodged. Whereas surgical data have shown acute MR reduction and positive remodelling 9 , the development of the percutaneous procedure has been stopped.

The complexity of the MV anatomy makes implantation of a percutaneous valve in the mitral position much more challenging than in an aortic position. However, despite potential difficulties, several transcatheter MV replacement technologies, transapical or transseptal, are currently in various stages of evaluation and development ${ }^{10}$.

\section{Conclusion}

The MV is a complex anatomical and functional structure. The development of transcatheter techniques for the treatment of MR has led to special attention being paid to the MV anatomy, since its assessment and understanding are crucial for patient selection and procedural success. Echocardiography is the main imaging modality used for MV evaluation and plays an essential role in the selection of patients.

\section{Conflict of interest statement}

The authors have no conflicts of interest to declare.

\section{References}

1. Nkomo VT, Gardin JM, Skelton TN, Gottdiener JS, Scott CG, Enriquez-Sarano M. Burden of valvular heart diseases: a population-based study. Lancet. 2006;368:1005-11.
2. Zamorano JL, González-Gómez A, Lancellotti P. Mitral valve anatomy: implications for transcatheter mitral valve interventions. EuroIntervention. 2014;10 Suppl U:U106-11.

3. Lancellotti P, Tribouilloy C, Hagendorff A, Popescu BA, Edvardsen T, Pierard LA, Badano L, Zamorano JL; Scientific Document Committee of the European Association of Cardiovascular Imaging. Recommendations for the echocardiographic assessment of native valvular regurgitation: an executive summary from the European Association of Cardiovascular Imaging. Eur Heart J Cardiovasc Imaging. 2013;14:611-44.

4. Nguyen TC, Itoh A, Carlhall CJ, Bothe W, Timek TA, Ennis DB, Oakes RA, Liang D, Daughters GT, Ingels NB Jr, Miller DC. The effect of pure mitral regurgitation on mitral annular geometry and three-dimensional saddle shape. J Thorac Cardiovasc Surg. 2008;136:557-65.

5. Schofer J, Siminiak T, Haude M, Herrman JP, Vainer J, Wu JC, Levy WC, Mauri L, Feldman T, Kwong RY, Kaye DM, Duffy SJ, Tübler T, Degen H, Brandt MC, Van Bibber R, Goldberg S, Reuter DG, Hoppe UC. Percutaneous mitral annuloplasty for functional mitral regurgitation: results of the CARILLON Mitral Annuloplasty Device European Union Study. Circulation. 2009; 120:326-33.

6. Choure AJ, Garcia MJ, Hesse B, Sevensma M, Maly G, Greenberg NL, Borzi L, Ellis S, Tuzcu EM, Kapadia SR. In vivo analysis of the anatomical relationship of coronary sinus to mitral annulus and left circumflex coronary artery using cardiac multidetector computed tomography: implications for percutaneous coronary sinus mitral annuloplasty. J Am Coll Cardiol. 2006;48: 1938-45.

7. Feldman T, Kar S, Rinaldi M, Fail P, Hermiller J, Smalling R, Whitlow PL, Gray W, Low R, Herrmann HC, Lim S, Foster E, Glower D; EVEREST Investigators. Percutaneous mitral repair with the MitraClip system: safety and midterm durability in the initial EVEREST (Endovascular Valve Edge-to-Edge REpair Study) cohort. J Am Coll Cardiol. 2009;54:686-94.

8. Zamorano JL, Fernández-Golfín C, González-Gómez A. Quantification of mitral regurgitation by echocardiography. Heart. 2015;101:146-54.

9. Grossi EA, Patel N, Woo YJ, Goldberg JD, Schwartz CF, Subramanian V, Feldman T, Bourge R, Baumgartner N, Genco C, Goldman S, Zenati M, Wolfe JA, Mishra YK, Trehan N, Mittal S, Shang S, Mortier TJ, Schweich CJ Jr; RESTOR-MV Study Group. Outcomes of the RESTOR-MV Trial (Randomized Evaluation of a Surgical Treatment for Off-Pump Repair of the Mitral Valve). J Am Coll Cardiol. 2010;56:1984-93.

10. Banai S, Verheye S, Cheung A, Schwartz M, Marko A, Lane R, Jolicoeur EM, Garceau P, Biner S, Tanguay JF, Edelman ER, White CJ. Transapical mitral implantation of the Tiara bioprosthesis: pre-clinical results. JACC Cardiovasc Interv. 2014; 7:154-62. 